\title{
Model-Based Systems Engineering Pathfinder: Informing the Next Steps
}

\author{
Karen J. Weiland \\ NASA Glenn Research Center \\ 21000 Brookpark Rd \\ Cleveland, $\mathrm{OH} 44135$ \\ 216-433-3623 \\ Karen.J.Weiland@nasa.gov
}

\author{
Jon Holladay \\ NASA Engineering and Safety Center \\ Hampton, VA 23681 \\ 256-345-7250 \\ jon.holladay@nasa.gov
}

This material is declared a work of the U.S. Government and is not subject to copyright protection in the United States. Approved for public release; distribution is unlimited.

\begin{abstract}
In 2016, the NASA Engineering and Safety Center established a model-based systems engineering (MBSE) Pathfinder. The primary motivations for establishing the MBSE Pathfinder were to advance the Agency's applications of MBSE and capture lessons-learned to inform the next steps. The MBSE Pathfinder had four teams working in parallel for eight months on different topics of interest to NASA. The teams were encouraged to learn, and use creativity and innovation in their system modeling. The results were captured via reports, webinars, and a knowledge capture meeting. The approach taken for the MBSE Pathfinder was very successful in providing a number of lessons-learned for NASA and for other organizations considering MBSE or pathfinder efforts, and in building a very strong and collaborative user community.
\end{abstract}

\section{Introduction}

At a NASA Systems Engineering Summit in the fall of 2015, a critical need was identified across the majority of the ten NASA field Centers to advance the Agency's applications of MBSE. Representatives expressed their desires to utilize 21st century systems engineering technology, tools, and methods more effectively across their diverse portfolio of programs, projects, and technological innovations.

In response to this need, the NASA Engineering and Safety Center (NESC) established an MBSE Pathfinder in January 2016. The MBSE Pathfinder was developed around three primary goals:

- $\quad$ Apply MBSE to complex NASA missions,

- $\quad$ Align MBSE across NASA Centers,

- Capture issues and opportunities for evaluating next steps.

The first goal focused on applying MBSE to understand how easily it could be deployed and usefully applied, while also producing examples that both technical and programmatic management could understand. The activity was not a pilot in that it was not testing out new ways of doing systems engineering, but a pathfinder. The true goal of the activity was capturing lessons-learned, both good and bad. Four areas were chosen that could be evaluated independently and represented portions of integrated and complex NASA missions. The subject areas were 1) mission architecture use and reuse for a humans-to-Mars campaign of missions; 2) additive manufacturing for rocket engine development, 3) mission element design of a Mars lander, and 4) mission flow shadowing of a sounding rocket project. The MBSE 
Pathfinder also expanded the use of a NASA Cloud infrastructure for software tools and a system modeling collaboration area.

The second goal focused on the cultural and technical challenge of aligning the MBSE community across NASA. NASA has many users of MBSE at its Centers, but until recently there was little coordination or guidance among these efforts at the Agency level. A previous agency-level activity had evaluated the digital architecture needed for MBSE and those results were available for reference. Both the Jet Propulsion Laboratory and the Goddard Space Flight Center had hosted workshops covering a broad range of related topics. Numerous other Centers had MBSE working groups and were in the process of developing plans for future engagement. In addition, roughly three dozen classes had been presented on MBSE and related topics at the different Centers. A solicitation for the MBSE Pathfinder was sent to all ten Centers, and participants were organized into virtual teams that were diverse in geographical location, discipline background, aerospace experience, and MBSE experience. This team environment fully challenged the implementation of MBSE, this time with an emphasis on culture so that lessons-learned could be maximized.

The third goal focused on capturing lessons-learned, so that next steps at the agency-level would be informed by hands-on experiences doing the work that was typical of real NASA missions.

The benefits of using MBSE were presented in a recent International Council on Systems Engineering publication (Miller, 2015) and are not discussed here.

\section{Approach}

The approach chosen for the MBSE Pathfinder was to solicit nominations for the teams in the four focus areas. Because the level of experience of the participants was not known during the planning period, it was decided to break apart the problem and focus on learning, creativity, and innovation in system modeling of an existing system or concept rather than learning how to do system modeling while at the same time defining a new system. The teams were asked to capture lessons-learned and recommendations for next steps along the way and at the end.

\section{Planning and Start-up}

Planning for the MBSE Pathfinder occurred over the fall of 2015 to outline the top-level objectives, schedule, resources and training, and topic areas. The activity was to begin in February and conclude in September 2016. The participants were to be provided introductory training, travel to the training and at least one face-to-face meeting, and access to a consistent set of tools. The Centers were asked to provide labor hours for the participants at $75 \%$ time. Desired qualities of a participant were experience or ability to learn system modeling; capable of working in a fast-paced, diverse multi-center culture; able to learn and communicate with the home Center; and ability to innovatively engage systems engineering implementation. The objectives were fairly broad in the initial call, so the four teams were asked to define detailed objectives, milestones, schedules, and deliverables in their work plans. The NASA Program Executive for the NASA Cloud agreed to provide information technology resources, software licenses for the MBSE Pathfinder, and personnel to support the activity.

The NASA Systems Engineering Technical Fellow issued a call in January 2016 for nominations from all NASA Centers. Placement of the nominees on the teams was done to promote diversity of home Center, technical area, and aerospace and system modeling experience, as shown in Table 1. Each of the teams had a lead and five to eight participants. The average NASA or aerospace experience level of each participant was 19 years, with a range 
of 4 to 40 years. Two of the team leads were very experienced and two had less than ten years. Over half of the participants had not applied MBSE to real missions. Summer students and interns also participated, and some participants worked on two or more teams. Several additional people served as the MBSE Pathfinder management team to provide leadership, implementation, and advice and guidance.

Table 1: MBSE Pathfinder Team Information

\begin{tabular}{|l|c|c|c|c|}
\hline & Team 1 & Team 2 & Team 3 & Team 4 \\
\hline \hline Number of participants & 8 & 6 & 8 & 5 \\
\hline Number of Centers & 7 & 5 & 5 & 4 \\
\hline Engineering disciplines & $\begin{array}{c}\text { aerospace } \\
\text { electrical } \\
\text { mechanical } \\
\text { software } \\
\text { systems }\end{array}$ & $\begin{array}{c}\text { aerospace } \\
\text { mechanical } \\
\text { systems }\end{array}$ & $\begin{array}{c}\text { electrical } \\
\text { mechanical } \\
\text { software } \\
\text { systems }\end{array}$ & $\begin{array}{c}\text { aerospace } \\
\text { mechanical } \\
\text { systems }\end{array}$ \\
\hline Aerospace experience & \multicolumn{5}{|l}{} \\
\hline 25+ years & 4 & 2 & 4 & 1 \\
\hline 11 to 25 years & 2 & 0 & 1 & 2 \\
\hline 0 to 10 years & 2 & 4 & 3 & 2 \\
\hline $\begin{array}{r}\text { Previous MBSE High } \\
\text { experience }\end{array}$ & 0 & 1 & 0 & 1 \\
\hline Ledium & 3 & 0 & 3 & 1 \\
\hline Low & 4 & 1 & 3 & 0 \\
\hline
\end{tabular}

A virtual kick-off teleconference in early February began the work in earnest. The kickoff covered the goals and objectives of the work and how it relates to systems engineering advancement at NASA, an overview of MBSE and the MBSE Pathfinder approach, and the schedule of events. The participants were given reading and video assignments and asked to begin working with their teams on drafting work plans.

The first face-to-face meeting occurred in late February at the Wallops Flight Facility (WFF). Since this was the first time most of the participants had met each other, several sessions were scheduled to promote team-building and preparation and review of the teams' work plans. The meeting included three days of introductory hands-on SysML training.

\section{Operating Rhythm}

All participants were invited to attend monthly teleconferences, which began in March. Each team presented highlights of their progress and could request assistance for issues. The team leads and MBSE Pathfinder management team also had a brief weekly teleconference.

Three face-to-face meetings were held; the first was the one mentioned above. The second meeting was a mid-term in May at the Johnson Space Center. This meeting served as a check point to see how the teams were doing, address issues, and prepare for the remaining months of work. The agenda featured sessions by participants on topics such as system and software engineering tools, model-based project management, model verification and validation, capturing stakeholder information, and the use of patterns and reference models. The teams presented overviews of their work to date and were given feedback and suggestions on both 
the good and the not-so-good. Time was allotted for the teams to work face-to-face to assess their progress and make updates to their work plans. The third meeting was for knowledge capture in September at the Langley Research Center. The agenda featured special topic sessions and open discussions on various aspects of MBSE and the MBSE Pathfinder, to draw out lessons-learned. The meeting concluded with summary presentations by each team, a summary presentation to key agency-level stakeholders, and recognition of the achievements of each participant.

Teams 2 and 4 each held a face-to-face meeting. Team 2 used their face-to-face meeting to work with each other in real-time, and to see test components. Team 4 had a Technical Interchange Meeting with the Sounding Rocket Program Office and the NASA Sounding Rocket Operations Contract (NSROC) customers to discuss and evaluate the modeling effort.

Each of the four teams produced a final report and summary presentation charts, and provided the system model(s). The Implementation Lead compiled the recommended next steps and grouped them into actionable areas for planning a follow-on effort.

\section{Resources for Modeling}

The NASA Office of Chief Information Officer hosted the systems engineering and collaboration software tools as part of the Agency Cloud provisioning pilot. The commercial software tools included multiple licenses of systems engineering software tools, a central model repository, a collaboration tool, a simulation toolkit, and data exchange tools with interfaces to a variety of other tools. In addition, the MBSE Pathfinder participants were able to use several plugins and modules that resided at NASA Centers.

The MBSE Pathfinder participants were provided with links to on-line webinars; several reference books on system modeling, MBSE, and SysML; and the introductory hands-on SysML class. The training instructors were retained as advisors and provided guidance to solve issues and gave feedback on the teams' models. Other advisors helped the teams throughout the remaining months. Many of the MBSE Pathfinder participants were experienced in MBSE or knew of knowledgeable people at their home Centers and shared their expertise.

\section{Results}

\section{Goals, Objectives, and Accomplishments}

Each of the four teams defined their specific goals and objectives for their work and stated them in their work plans. Some goals and objectives were common, and fell into the general categories of construct a system model by either using existing information or shadowing an existing project, generate technical review products, investigate import and export capabilities and interactions with physics-based tools, and investigate model reuse. All the teams had significant accomplishments and findings.

The teams focused on different levels of system architectures ranging from campaign and mission to subsystems to construct a system model for their topic area. Team 1 modeled a campaign and mission architecture based on a published campaign architecture for human exploration of Mars (Arney, 2015). Team 2 modeled the new development of a liquid rocket engine. The elements modeled included the needs, goals, and objectives; constraints; hierarchical diagrams; use cases; engine requirements; and data management. Team 3 modeled systems and subsystems typical for a spacecraft, and studied system and physical decomposition and interfaces. Team 4 created an interconnected model of the Multiple User Suborbital Instrument Carrier (MUSIC) sounding rocket project. The team used the Sounding 
Rocket Design Review requirements and MUSIC mission technical review data package as their primary information sources.

The teams looked at different parts of a project lifecycle and used the system model to generate technical products for different reviews. Team 1 looked at a Program System Requirements Review and Program System Definition Review, and the project-level Mission Concept Review and a System Requirements Review as defined in NASA Procedural Requirements (NPR) 7120.5 and NPR 7123.1 (NASA, 2010; NASA, 2015). Team 2 began work towards a System Design Review that would occur in the future. They presented the engine system and component concepts and looked at ways to ensure that the system requirements were developed and communicated to the system team. Team 3 generated a subset of the technical review package for a Preliminary Design Review. Team 4 modeled the content in a sounding rocket mission Design Review package and used the system model to generate the information in a way that the stakeholders would find acceptable.

The teams attempted to perform data import, data export, and data exchange with other software tools such as those for office productivity, computer-aided design (CAD), and simulation. This was especially desirable for performing trade studies and for collaboration with other team members. Team 1 performed constraint analyses for impacts associated with changes in launch dates for cargo missions and mass limits for each launch. Team 2 began work to use the system model along with CAD models to automatically update requirements and specifications for changes and notify all affected system and component leads. They also wanted to use the system model to track interface requirements as the design evolved. Team 3 mapped the blocks in their architecture components to CAD model elements to allow properties to be visible and up-to-date. They also used the system model with simulation software. Team 4 performed import and export of requirements.

All of the teams emphasized the creation of a model that had areas of reuse for campaign, missions, systems, or subsystems. The teams looked at developing templates and model element libraries. Team 1 developed numerous models for a Mars campaign and a mission to take in-situ resource utilization (ISRU) cargo to the Mars surface. They developed a model library for the areas of behavior, structure, and requirements, and the relationships among them. Team 2 began efforts in this area and constructed a model of common engine parts. Team 3 developed and used a component library of spacecraft subsystem models, a library of terminology, and interface definitions. Team 4 developed a reusable model library of commonly-used sounding rocket spacecraft and enabling ground and support systems parts and model elements. They then used the generic model elements, including the system reference architectures and decomposition patterns, and addressed the specific payload and operational requirements for the MUSIC mission. All of the completed libraries were delivered at the end of the work period and are available for reuse for follow-on work.

\section{Innovations}

All four teams were encouraged to be creative and innovative in their system modeling. Teams had innovations in work processes, model organization, and analyses in addition to the accomplishments previously mentioned.

Several teams used different approaches for their modeling efforts. After several months of work using a traditional project schedule, Team 1 switched to an agile systems engineering approach. This was unusual in that an agile approach is usually associated with co-located teams, and this was a virtual team. They used sprints and spikes to complete five sprint cycles, with planning and retrospectives, with a focus on essentials and quick accommodation of 
changes in team member availability. After several successful sprints with a very limited number of work objectives, Team 1 changed their approach to put forth efforts on multiple work objectives in the sprint. Even though agile principles for a sprint include doing work, or at least some work, on all aspects of the work plan, the team members felt that there were too many work activities for those particular sprints. The team noted that both approaches could be considered when planning. The team also used a buddy system and pairwise assignments approach to increase team member engagement levels and team communications about the modeling work.

In order to mimic project level execution, Team 3 organized themselves into three product groups: infrastructure, system engineering and integration, and engineering (subsystems). They allowed each subsystem team member to have an individual project with shared modules that other projects could use. The system model used the concept of libraries to maintain consistency. Then at the system level, the workflow was planned to ensure proper integration by the system integrator. This arrangement successfully allowed team members to work both collaboratively and independently as needed.

Several teams organized their models to contain both the system being modeled and other aspects of interest. Team 1 used a top-level structure in their model that paralleled their two focus areas: 1) model a campaign of missions to establish a human presence on Mars, and 2) learning, creativity, and innovation in system modeling. The top level of the model had two packages: a system model of the Mars campaign and constituent missions, and a project management package for the modeling effort itself. These packages provided a place to model the system-of-interest and to manage the modeling effort, capture lessons-learned, and show how the MBSE Pathfinder deliverables were being met. Team 4 had two models: an engineering model of the sounding rocket mission and a program model of the Sounding Rocket Program, its project lifecycle, and the measures of success used to determine return on investment. The team partnered with personnel for the Department of Defense to create the program model. This program model will allow the team to track Sounding Rocket Program cost, schedule, risk and technical performance before and after MBSE implementation. The Model Based Sounding Rocket project will be one of the first studies of its kind to utilize MBSE for program and project management and to measure MBSE's return on investment. Team 4 also had several packages in their model for communicating their models, such as "Presentations" and "Model Showcase." The Presentation package contained links to elements in the model that were used during presentations, and allowed easier transition between slides and showing information directly from the model. The Model Showcase was used to organize how the model would be presented to the MBSE Pathfinder colleagues at the monthly telecons.

Several teams performed parametric analyses that extended beyond those typically done in MBSE. Team 1 performed sensitivity analyses at the campaign level for two factors (mass limits for a particular launch, and slips or delays in launch dates). Team 2 developed several innovative analyses. The first was a way to store numeric requirement values. Since SysML requirements are intended to be text strings, a new property was created and associated with all requirements. Although the values for the property were entered manually, this did provide a way to store the values for use during automatic requirements verification. The second analysis was for the export and evaluation of instances. During the rocket engine design process, multiple properties can change as the design evolves, so there is a need to auto-generate up-todate requirements and performance specifications. The team wrote custom scripts to show the properties of an instance in a readable fashion and evaluate an instance against the requirements for compliance. Prior to starting this analysis, the team wrote a custom script to import and store engine power balance data in the system model. 


\section{Benefits to NASA}

All of NASA benefited from the MBSE Pathfinder at the Agency level and at each of the Centers. The MBSE Pathfinder participants became a trained and experienced cohort of 30+ people, and are now a "go-to" resource for the Agency and their Centers.

A series of webinars hosted by the NESC occurred in July, September, and October of 2016. The first four webinars featured a topic of interest for future space exploration and an MBSE response. A NASA technical expert presented the technical challenges of the topic, and an MBSE Pathfinder participant presented a response that highlighted work being done by one of the teams about the topic. The fifth webinar featured the NASA Cloud deployment and a summary of the MBSE Pathfinder effort. The webinars were open to all NASA employees, and were recorded and placed on-line in the NESC Academy. In addition, all the MBSE Pathfinder teams submitted final reports that are available for use in making future plans at the agencylevel. Several other presentations at the agency-level were made, most notably at the 2016 NASA Cost Symposium.

In addition to the NESC webinars, each team provided direct benefits to their focus area. Team 4 demonstrated MBSE benefit to their focus area in two additional ways. The first was through the modeling of the current design review documentation and finding information that was missing, such as a clear definition of the interfaces between the experiment and the sounding rocket, and inconsistent such as in test plans. The second way was through the examination of standard operating processes. The use of MBSE could help with information transfer among the NSROC engineers and its external stakeholders such as the experimenter, launch range, safety, and the Sounding Rocket Program Office.

The MBSE Pathfinder served as the first effort to provision software in the Cloud operating environment for agency-wide use and collaboration as part of the Cloud Provisioning Pilot that is sponsored by the Office of Chief Engineer and hosted by the NASA Headquarters Office of the Chief Information Officer. The establishment of the server environment and software installation went well, though some interaction between Cloud host and the software provider was required to match the tool with NASA protocols and settings. After the initial set-up, little interaction with the software provider was required. Software updates and license key renewals went smoothly and were incorporated with no disruption in service. During the course of the MBSE Pathfinder, the software was integrated into the NASA cybersecurity logon authentication system. This required considerable effort on the part of Agency cybersecurity officers and the software vendor. Given the dynamic nature of the cybersecurity environment for all software, this issue will require continuous monitoring and remains a topic to be addressed in evaluating other tools for cloud operations.

Many presentations to NASA Centers, engineering, and other managers were given. An increase in Center and other management interest and support was evidenced by requests for presentations about the MBSE Pathfinder, inquiries about use on new programs and projects, encouragement for discussions on potential collaboration areas, and support for training courses.

All the NASA Centers that had a participant on the MBSE Pathfinder had an immediate and significant increase in MBSE awareness and involvement of their engineering staff. Participants revived or increased participation in MBSE communities of practice, working groups, and learning groups, and created a new working group. Participants gave presentations to peers, conducted knowledge exchanges, and shared information about training resources. They mentored colleagues, supported intern training, and shared their knowledge about the 
work they were doing and its benefits. Participants began modeling on their "day" job projects. Finally, at least one project proposal related to MBSE that had multi-center participation was prepared.

\section{Learning to Model}

Many forms of learning were used during the MBSE Pathfinder, both within and outside the MBSE Pathfinder team structure. Participants performed learning on the job as part of their team work; received hands-on training, consultation services, and feedback on their modeling products; and reviewed and reused other teams' models. Several demonstrations and "show and tell" sessions were given by teams and team members to their MBSE Pathfinder colleagues. Participants attended Agency webinars on MBSE Pathfinder-related topics and webinars from tool vendors. Participants also interacted with colleagues at their home Centers for advice and guidance.

The participants learned how to perform systems engineering in a virtual team and model-based environment. Specific topics related to modeling were learning the SysML language and a tool suite; how to put content from documents and graphics into a system model, combine models, use library components, and import and export information; and the importance of modeling with a purpose. Learning areas emphasized the systems engineering processes, the team environment, and stakeholder interactions. Participants learned how to generate documents and technical review package contents, how to work in a collaborative area with virtual team members and with multiple modelers on one project, how to use a collaboration area for reviews, and how to display information for the stakeholders and organize a model so as to present from a model.

All the MBSE Pathfinder participants were asked to submit a self-assessment of their MBSE skills at the start, mid-point, and end. The ratings scale was from 1 to 10 , with 1 being none, 5 being a competent practitioner in common aspects, and a 10 being an expert practitioner. The responses were grouped together for all the respondents into four categories and are shown in Figure 1. The results shown here are for those participants who completed all three selfassessments. At the start, most participants had no or low skills. At the mid-point roughly two and a half months after the initial WFF training and team work sessions, a significant number moved from "None" to "Low" and a few moved from "Low" to "Medium." At the end point, which occurred seven months after the start, all participants had improved skills, with twothirds being at a "Medium" or "High" skill level.

\section{Difficulties}

Each of the four teams encountered difficulties throughout their work. This was expected to occur as the teams were newly-formed and working mostly in a virtual environment, and many of the participants were novice modelers.

The teams collected metrics on the amount of time spent on the MBSE Pathfinder activity. The teams also tracked hours spent in meetings, including working meetings, and hours with advisors and coaches. Since most individuals were not full time on the MBSE Pathfinder, many were sometimes unexpectedly unavailable due to being asked to work higher priority reviews and projects at the Centers. The time periods varied from weeks to several months. Some teams noted that a few people were able to work slightly more hours that made up for others working fewer hours. The amount of time available collectively was slightly less than planned; for Team 2 it was about half what was planned. As the time progressed, some teams had team member turnover and adjusted roles and tasks. The reduction in time and lack of efficiency in modeling led to re-scoping of efforts and some frustration about the lack of progress. 


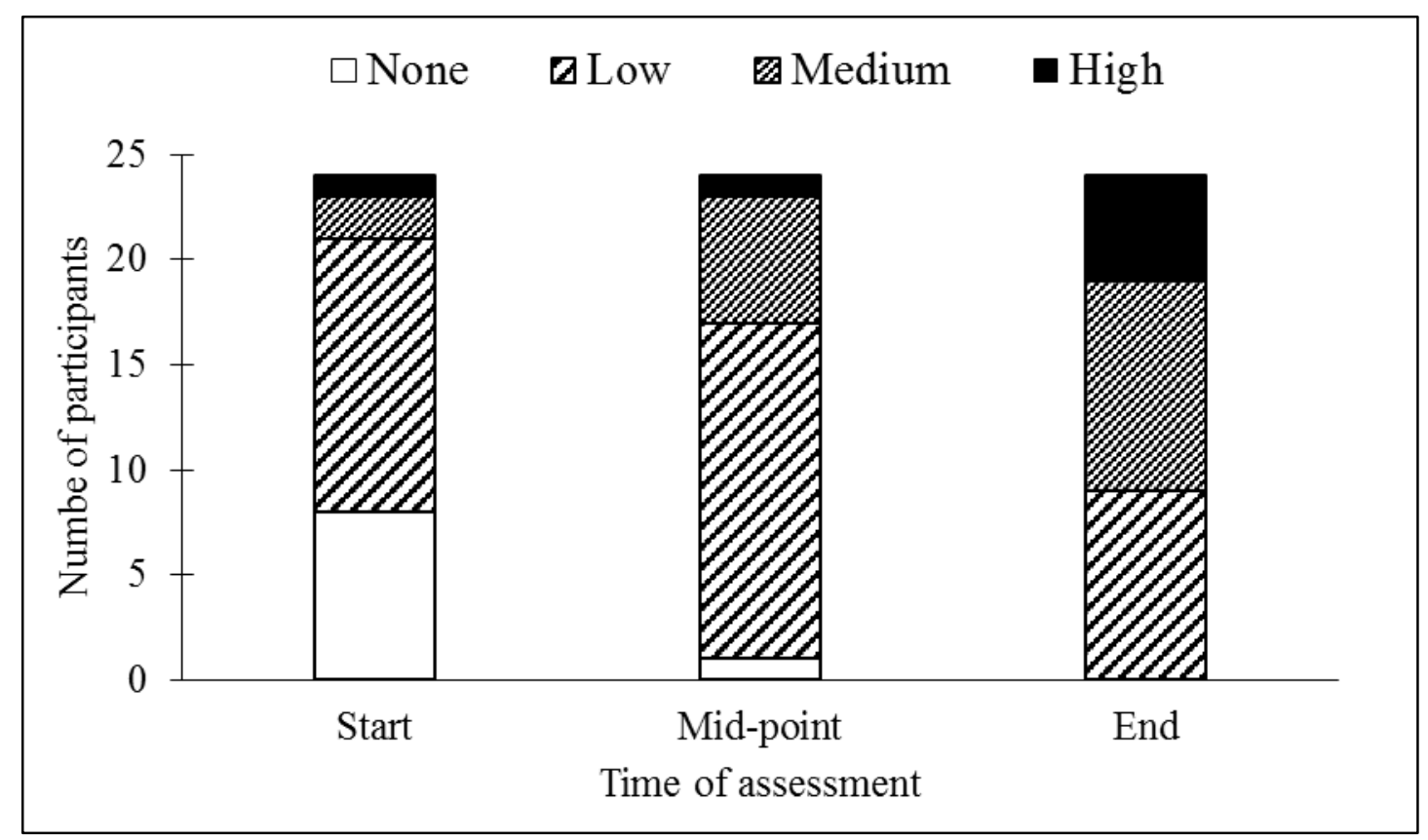

Figure 1. MBSE Skills Self-Assessment Results

Some of the teams had clear ideas about what they wanted to accomplish but were not able to perform the desired functions in the tools. This may have been due to unfamiliarity with the tools and SysML, a limitation in SysML, or how SysML was implemented in the tools. Many users noted that the software tools had a complex user interface. They noted that regardless of what tool was used, all were a significant change from previous experience. The users noted that novices needed either constant guidance or examples, or they made many mistakes before discovering a solution. Most of the teams observed that it was difficult to learn how to model while learning how to set up a model structure. They experienced information overload, with too much information in too short a time to figure out what to do first. As a result, many of the teams began modeling and at a later time rebuilt their models or noted that there were issues. In addition, the teams were on their own to come up with their own best practices for modeling methodologies.

Document and table generation was found to be difficult. Generating a document into a format that was familiar to the stakeholder required either scripting or manually manipulating the final output after using built-in templates. Other users noted difficulties with parametric analyses and tried several tools or wrote custom scripts to find a solution. Some difficulties were selfinflicted in that the model was not structured properly for the analysis. Data import, export, and exchange worked for simple cases, but anything more complicated, such as selecting the parameters to be exchanged or doing analyses of a system through time, required custom scripts or additional modeling elements or tools.

All the teams experienced issues with collaboration while working on the model. Even though all the teams communicated well as a team and among individuals, issues arose with model change control and reconciling differences. Some teams had occurrences where the ownership of model elements was not clearly assigned, so conflicts arose with the locking and unlocking of model elements during and after editing. Team 1 used a "sandbox" approach; Team 3 used separate projects with shared modules that were later integrated into a master project by a system integrator. Some difficulties in change control were due to the different approach of tracking modeling changes compared to software code changes. The system modeling tool 
tracked changes both in model elements and in diagrams, so it was difficult to detect what were the essential changes.

\section{Knowledge Capture}

Near the end of the MBSE Pathfinder, a knowledge capture meeting was held to discuss the past eight months of work. It was the final face-to-face meeting and brought together participants from the four teams, the NASA Cloud infrastructure, and management to share their knowledge about their experiences and discuss the next steps forward for an individual, Center, or the Agency. The participants had special topic sessions related to MBSE and a round table to discuss what to do about MBSE at the Agency level. Each participant had a different perspective to offer based on experience level, team assignment, and role on the team. The MBSE Pathfinder teams were intentionally set up to be diverse in experience and multi-center so as to get the different perspectives while the teams were working.

The special topic sessions lasted an hour and a half each with three running in parallel at a time. Each session had a leader and a recorder and were given a topic and ideas of what to discuss. The following day, fifteen minute reports on each topic were presented to the entire group. An open discussion in a round table format then occurred. Table 2 lists the session titles and the discussion areas, and has a summary of the comments and recommendations from the sessions. Specific recommendations that would improve the adoption and use of MBSE by individuals, projects, Centers, and Agency were given to agency-level management. The final reports from each team also contained recommended next steps.

The Agency-level next steps fell into six major areas: 1) establish an Agency community of practice with an online collaboration space and libraries, 2) provide handbooks, training, seminars, and other information for getting started in MBSE, 3) determine what software tools are used and what are needed, and identify solutions for future NASA needs, 4) give guidance on how to use MBSE with NASA's systems engineering processes, such as how to produce milestone review products, 5) architect the NASA systems and infrastructure for MBSE, and 6) demonstrate the value of MBSE and gain the interest of projects and external partners.

As a result of the Knowledge Capture information and recommendations, a pilot effort for a NASA MBSE Community of Practice (CoP) was established and the MBSE Pathfinder was set up to run for a second year. Both of these efforts started in November 2016.

The CoP is piloting a knowledge repository and online collaboration area that will facilitate and grow the efforts of MBSE practitioners across NASA by disseminating best practices and lessons-learned and promoting model reuse and common MBSE practices.

The MBSE Pathfinder objectives for the second year are to demonstrate and communicate value to the projects and NASA Centers. The participants are implementing lessons-learned and emphasizing the development of systems engineering products across the life-cycle and integrated with various analytical tools, such as computer-aided design tools and loads analysis tools. The five mission topic areas are in-situ resource utilization trade studies, in-space habitat element requirements and design, engine requirement compliance and test configuration, launch vehicle payload adapter structure design and build, and sounding rocket mission flow shadowing. In addition, a small team is collaborating with the first two teams to do cross-team analyses. Finally, a cross-cutting themes team is collecting content from the MBSE Pathfinder and other sources that is useful for all the teams and beyond. Initial efforts are on a handbook, a glossary, and examining options for MBSE in the future. This content will be provided to the NASA MBSE Community of Practice for their knowledge repository. 
Table 2: Knowledge Capture Meeting Special Sessions

\begin{tabular}{|c|c|c|}
\hline Session Title & Discussion Areas & Comments and Recommendations \\
\hline $\begin{array}{l}\text { Lessons- } \\
\text { Learned about } \\
\text { this Pathfinder }\end{array}$ & $\begin{array}{l}\text { What to continue or } \\
\text { change for a follow-on } \\
\text { and what focus areas } \\
\text { of interest should be } \\
\text { continued or changed }\end{array}$ & $\begin{array}{l}\text { - Focus was on learning how to model a } \\
\text { system, not necessarily doing SE } \\
\text { - Suggestions for follow-on work } \\
\text { - Model more of the life-cycle of a product } \\
\text { - Link models from different teams } \\
\text { - Embed an expert on each team }\end{array}$ \\
\hline $\begin{array}{l}\text { How to Do } \\
\text { Systems } \\
\text { Engineering } \\
\text { with MBSE }\end{array}$ & $\begin{array}{l}\text { What changes to } \\
\text { NASA processes or } \\
\text { NPR "shall" statements } \\
\text { needed to be made and } \\
\text { if any areas were ready } \\
\text { to convert from } \\
\text { document-centric to } \\
\text { model-centric }\end{array}$ & $\begin{array}{l}\text { - Too early to standardize on a specific toolset } \\
\text { - General acceptance of SysML for MBSE } \\
\text { - Start the adoption now, in a thoughtful and } \\
\text { incremental way } \\
\text { - Need a mapping from documented-based } \\
\text { systems engineering artifacts to MBSE } \\
\text { products } \\
\text { - Configuration Management is critical to a } \\
\text { model-based approach }\end{array}$ \\
\hline $\begin{array}{l}\text { Learning to } \\
\text { Model }\end{array}$ & $\begin{array}{l}\text { Types of training, } \\
\text { mentors, on-the-job } \\
\text { training, aids, and } \\
\text { examples }\end{array}$ & $\begin{array}{l}\text { - Team experience ranged from novices to } \\
\text { experts } \\
\text { - A lot to learn - language, tool chain, team } \\
\text { work processes } \\
\text { - Developing a model from within a familiar } \\
\text { domain helps } \\
\text { - Focus on a particular project: have a } \\
\text { problem to solve } \\
\text { - Find a mentor to help }\end{array}$ \\
\hline $\begin{array}{l}\text { NASA } \\
\text { Infrastructure }\end{array}$ & $\begin{array}{l}\text { Collaboration } \\
\text { environment, access to } \\
\text { software and licenses, } \\
\text { and access to } \\
\text { knowledge }\end{array}$ & $\begin{array}{l}\text { - Recommend a NASA-wide MBSE wiki to } \\
\text { facilitate communication and socialization } \\
\text { => glossary, best practices, etc. } \\
\text { - Continue to grow the NASA Cloud => more } \\
\text { access and more users } \\
\text { - Establish a pilot team to engineer the MBSE } \\
\text { ecosystem }\end{array}$ \\
\hline $\begin{array}{l}\text { MBSE with } \\
\text { Other Areas }\end{array}$ & $\begin{array}{l}\text { Configuration } \\
\text { management, fault } \\
\text { management, project } \\
\text { management, and } \\
\text { physics based models }\end{array}$ & $\begin{array}{l}\text { - Involves data transformation, presentation, } \\
\text { analysis, and multiple levels of detail } \\
\text { - Consider data as the "single source of truth" } \\
\text { - Develop criteria to decide what data is in a } \\
\text { SysML model and what is in discipline areas } \\
\text { - Develop profiles of NASA standards }\end{array}$ \\
\hline $\begin{array}{l}\text { Acceptance of } \\
\text { MBSE }\end{array}$ & $\begin{array}{l}\text { Acceptance of MBSE } \\
\text { with line management, } \\
\text { project management, } \\
\text { systems engineering } \\
\text { peers, peers in other } \\
\text { disciplines, and review } \\
\text { boards }\end{array}$ & $\begin{array}{l}\text { - Scattered use at centers with small successes, } \\
\text { not a cohesive story } \\
\text { - Identify a champion at each Center to tie } \\
\text { stories together } \\
\text { - Use stakeholder analysis to drive } \\
\text { implementation priorities }\end{array}$ \\
\hline
\end{tabular}




\section{Analysis}

The approach and results described above clearly show that the MBSE Pathfinder was very successful. Table 3 lists the top-level MBSE Pathfinder goals and objectives established at its on-set and summarizes the evidence for this assessment. The amount and type of information obtained from the MBSE Pathfinder validated many commonly-held ideas and impressions about MBSE, and provided new input.

\section{Factors for Success}

The success of the MBSE Pathfinder work in 2016 did not happen by chance; previous NASA attempts in this area lacked the type, quantity, and significance of accomplishments. So what was different? Three key areas were management support, a focus on quick, real-world experiences, and active involvement of both experienced and expert personnel as leaders and team members.

The MBSE Pathfinder had support from all levels of management. At the agency-level, the NESC sponsored the work and actively voiced their commitment to the work in multiple venues. Information about the work was presented to different management groups, agencylevel peers, and to the discipline technical fellows. The Office of Chief Engineer Special Projects Program Executive was interested in using the MBSE Pathfinder to demonstrate the ability to expand the NASA Cloud to provide common, floating licenses across Centers, and contributed personnel, information technology resources, and traveled to participate in and present at the face-to-face meetings. Center management expressed their support by their nominations of talented people, all of whom were interested and willing to learn. All levels encouraged a culture of open discussion of issues and challenges.

The MBSE Pathfinder had a management team that worked to keep the effort on track. An Implementation Lead kept the teams organized and focused on their efforts; this required about 30 to $50 \%$ of work time for the past year. The management team scheduled and organized the face-to-face meetings, and found modeling experts when the teams were struggling.

The approach to the MBSE Pathfinder allowed for success to occur even when the modeling effort and results did not meet all the objectives set by the teams. The focus was on learning and communicating what was learned instead of making a perfect model. The teams were allowed to try out different modeling approaches to see what worked and what didn't work. And doing an eight month effort allowed for results to be fed back quickly, instead of waiting for several years to pass before seeing results. Using real-world NASA missions provided value for those focus areas.

Active involvement of experienced and expert personnel were a key factor for success, but in unexpected ways. Two of the team leads had about 30 years of experience and two had less than 10. And several of the team leads were not in systems engineering organizations, but were in areas such as software independent verification and validation or propulsion engineering. These results indicate that long years of experience, especially in systems engineering, may not be a strong indicator of success for team leadership. For the modeling effort, some experts had only a few years and some had 25+ years of aerospace experience, and all were effective. The level of comfort with and ability to use new software technology appeared to depend more on the individual's abilities and interests, rather than some minimum years of experience. 
Table 3: Assessment of MBSE Pathfinder Success

\begin{tabular}{|c|c|}
\hline Goal or Objective & Evidence of Success \\
\hline Multi-center collaboration. & $\begin{array}{l}\text { - Eight NASA Centers and JPL participated. } \\
\text { - Each team had participants from four or more Centers. }\end{array}$ \\
\hline $\begin{array}{l}\text { Recognize champions and } \\
\text { users of MBSE within } \\
\text { NASA. }\end{array}$ & $\begin{array}{l}\text { - Many participants had some experience with MBSE. } \\
\text { - Participants identified experts and other resources at their } \\
\text { Centers. }\end{array}$ \\
\hline $\begin{array}{l}\text { Understand opportunity } \\
\text { and difficulty for } \\
\text { implementing an agency- } \\
\text { wide integrated approach. }\end{array}$ & $\begin{array}{l}\text { - Participants used the NASA Cloud for software resources. } \\
\text { - Eight months of experience increased understanding; } \\
\text { opportunities and difficulties were in the final reports. } \\
\text { - Participants noted that agency-wide collaboration area was } \\
\text { highly desirable. }\end{array}$ \\
\hline $\begin{array}{l}\text { Develop expert capability } \\
\text { (be a smart buyer) that can } \\
\text { assist in developing a } \\
\text { systems engineering vision } \\
\text { and next steps. }\end{array}$ & $\begin{array}{l}\text { - 30+ participants on the teams. } \\
\text { - Multiple topics in MBSE. } \\
\text { - Topics were explored collectively in sufficient depth to } \\
\text { understand the area. }\end{array}$ \\
\hline $\begin{array}{l}\text { Examine reuse of models } \\
\text { and data. }\end{array}$ & $\begin{array}{l}\text { Teams developed reference architectures, libraries, } \\
\text { templates, and other reusable items. } \\
\text { - Teams examined use of models for data analysis and } \\
\text { storing data associated with model elements such as value } \\
\text { properties. }\end{array}$ \\
\hline $\begin{array}{l}\text { Apply MBSE to real } \\
\text { NASA issues. }\end{array}$ & $\begin{array}{l}\text { - The four focus areas were related to NASA missions: } \\
\text { Mars architecture campaign, rocket engine development, } \\
\text { Mars lander development, and sounding rocket program. }\end{array}$ \\
\hline $\begin{array}{l}\text { Develop aligned capability } \\
\text { and community across the } \\
\text { Centers. }\end{array}$ & $\begin{array}{l}\text { - Grew the NASA Cloud and its resources were available to } \\
\text { all participants. } \\
\text { - Communities of practice at several Centers had increased } \\
\text { participation or were reinvigorated. }\end{array}$ \\
\hline $\begin{array}{l}\text { Get all Centers to at least } \\
\text { the same minimum level. }\end{array}$ & $\begin{array}{l}\text { - Almost all Centers had one or more participants. } \\
\text { - Initial training class for all participants provided a } \\
\text { common starting point. } \\
\text { - NESC Webinars were open to anyone at NASA. }\end{array}$ \\
\hline $\begin{array}{l}\text { Provide an opportunity to } \\
\text { participate in an agency- } \\
\text { level activity and change } \\
\text { the culture. }\end{array}$ & $\begin{array}{l}\text { - NESC sponsored the activity. } \\
\text { - Final Reports were analyzed by Office of Chief Engineer } \\
\text { leadership for forward action plans. }\end{array}$ \\
\hline $\begin{array}{l}\text { Capture issues and } \\
\text { opportunities (lessons- } \\
\text { learned). }\end{array}$ & $\begin{array}{l}\text { - Monthly team virtual meetings. } \\
\text { - Knowledge Capture meeting. } \\
\text { - Final Reports. }\end{array}$ \\
\hline
\end{tabular}

\section{Inter-center Collaboration}

The MBSE Pathfinder teams demonstrated that inter-center collaboration worked, but it did require conscious and concerted efforts. In most cases, only one or two individuals from each Center were on a team. Most multi-center NASA project work has a lead Center and other participating Centers are given responsibility for a work package. Those Centers typically assign a team to the work package. It is not typical for individuals to be embedded on another 
Center's team. However, NASA management is looking at using engineering talent regardless of Center affiliation. Although most of the teams struggled to work in the virtual environment, they accomplished a significant amount in this new way of doing business. In addition, the participants now have many personal contacts at other NASA Centers in addition to those at their home Centers.

Cultural issues were addressed at all three face-to-face meetings and deliberate efforts were made to foster team morale. At the initial meeting, the training was interleaved with team work sessions to allow the teams to work face-to-face so that the teams could then work better over the remaining months in a virtual environment.

\section{"Reach" of the MBSE Pathfinder}

The extent to which the MBSE Pathfinder touched or effected the NASA workforce was estimated by examining how the participants interacted with colleagues at other NASA Centers and across the Agency. Over twenty presentations were made at the Centers to many different groups along with the five NESC webinars, and six Center MBSE working groups were improved or revitalized. New collaborations were formed with personnel in the Office of the Chief Financial Officer and the Office of Safety and Missions Assurance. A rough estimate is that over 600 people at NASA have already been directly informed about the MBSE Pathfinder work.

\section{Conclusions}

The MBSE Pathfinder is an outstanding example of what teams can do when they are empowered and challenged to attempt what some considered impossible. From the Agency perspective, the MBSE Pathfinder represented a very innovative approach to building systems engineering capability. It was focused very simply on one of the Agency's top technical risks, "to utilize 21 st century systems engineering technology, tools, and methods more effectively across their diverse portfolio of programs, projects, and technological innovations," and provided a rich harvest of lessons-learned on the engagement of MBSE in a rapid, efficient, and results-driven manner.

The approach used here could be used by other organizations considering MBSE or pathfinder efforts. Systems-of-interest to be modeled were selected from top priorities within the NASA Strategic Plan (NASA, 2014) and represented current and future states of complex systems. These included utilization of Mars in-situ resources and future state advanced manufacturing, as well as the simulation of mission elements and integration flow. Teams were chosen and populated to maximize diversity: culturally, geographically, and technically. The virtual environment used for modeling and collaboration also reflected the most modern situation possible, leveraging and expanding the NASA Cloud as well as software licensing and common storage and sharing of models.

At the completion of the eight month effort, digital models for the four systems-of-interest were developed to exercise various aspects of systems engineering, such as system architecting, system analysis, and milestone reviews. As a group, the participants significantly increased their MBSE skills. Technical, organizational, and cultural aspects were considered in the approach and evaluation of results. Over fifty lessons-learned based on in-depth, hands-on experience were captured to guide next steps for the implementation of MBSE at NASA. Many hundreds of people at NASA were directly touched by the effort from a relatively small number of direct participants. A very strong and collaborative community was established that is not only a NASA resource, but a national and international resource. 


\section{References}

Arney, D., Jones, C., Klovstad, J., Komar, D.R., Earle, K., Moses, R., Bushnell, D., and Shyface, H., 2015, "Sustaining Human Presence on Mars Using ISRU and a Reusable Lander," AIAA SPACE 2015 Conference and Exposition, AIAA 2015-4479. Pasadena, CA (US).

Miller, W. (ed.), 2015, INSIGHT, 18 (2), International Council on Systems Engineering, San Diego, CA (US).

National Aeronautics and Space Administration, 2010, NASA Procedural Requirements 7120.5E, "NASA Space Flight Program and Project Management Requirements w/Changes 1-12," NASA Office of the Chief Engineer. Washington, DC (US).

_, 2014, "NASA Strategic Plan," NASA Headquarters, NP-2014-01-964-HQ. Washington, DC (US).

, 2015, NASA Procedural Requirements 7123.1B w/ Ch3, "NASA Systems Engineering Processes and Requirements," NASA Office of the Chief Engineer. Washington, DC (US).

\section{Biography}

Karen Weiland is a Systems Engineer at the NASA Glenn Research Center. She received a B.S. in Chemistry from the University of Notre Dame and a Ph.D. in Chemistry from the University of Wisconsin - Madison. Her career started with microgravity combustion diagnostics research and as a project scientist for spaceflight experiments on the Space Shuttle and the International Space Station. She performed systems engineering on spaceflight projects, most recently for the Spacecraft Fire Safety Demonstration (Saffire) project. She is currently working at the Center and Agency levels to improve systems engineering capabilities across NASA.

Jon Holladay currently serves as the NASA Technical Fellow for Systems Engineering. His career encompasses both science and human space flight missions ranging from Hubble Space Telescope to the International Space Station and Space Launch System. He provided flight certification and led flight operations for five Multi-Purpose Logistics Module missions. He was Chief Engineer for the Marshall Space Flight Center Flight Programs and Projects Office, Ares V launch vehicle development and Space Launch System evolvability. Mr. Holladay received both his bachelor's and master's degrees in mechanical engineering from The University of Alabama. 